\title{
DIFERENCIAS DE GÉNERO EN LA MOTIVACIÓN Y EN LOS MÉTODOS DE DISPOSICIÓN DE PRODUCTOS EN UN ENTORNO COLECTIVISTA DE ALTA MASCULINIDAD
}

\author{
GENDER DIFFERENCES IN MOTIVATION AND PRODUCT DISPOSAL METHODS IN
} A HIGH MASCULINITY COLLECTIVISTIC ENVIRONMENT

\section{Jorge Cruz Cárdenas}

Doutor em Administração de Empresas - Marketing Management Professor e pesquisador no Centro de Investigación, Innovación y Desarrollo pela Universidad Tecnológica Indoamérica - Equador E-mail: jorgecruzc@yahoo.com (Equador) 


\title{
DIFERENCIAS DE GÉNERO EN LA MOTIVACIÓN Y EN LOS MÉTODOS DE DISPOSICIÓN DE PRODUCTOS EN UN ENTORNO COLECTIVISTA DE ALTA MASCULINIDAD
}

\section{RESUMEN}

La disposición de los productos es una complicada forma de comportamiento del consumidor que implica que este deje de usar un producto que aún conserva su capacidad de funcionamiento. El presente estudio se orientó a establecer las diferencias de género en dos etapas del proceso de disposición: La motivación y la decisión al respecto del método de disposición. Mediante una encuesta aplicada a 414 personas, en Quito, Ecuador, se pudo establecer una relación muy importante entre las variables de interés y género, relación a partir del cual se discuten sus implicaciones.

Palabras clave: Disposición de productos, diferencias de género, comportamiento del consumidor.

\section{GENDER DIFFERENCES IN MOTIVATION AND PRODUCT DISPOSAL METHODS IN A HIGH MASCULINITY COLLECTIVISTIC ENVIRONMENT}

\begin{abstract}
The disposal of products describes the behavior of consumers who stop using a product that still maintains working capacity. The present study was oriented to examine gender differences within two stages of the disposal process: the motivation and the decision regarding the method of disposal. By means of a survey applied to a sample of 414 individuals in Quito, Ecuador, a significant relationship between personal interests and gender variables was established. The implications of this relationship are discussed.
\end{abstract}

Keywords: Product disposal, gender differences, consumer behavior.

Revista Brasileira de Marketing - REMark, São Paulo, v. 12, n. 2, p. 158-179, abr./jun. 2013. 


\section{INTRODUCCIÓN}

A lo largo de las décadas, la investigación sobre el comportamiento del consumidor ha puesto fuerte énfasis en el proceso de decisión de la compra, y en aspectos de la post compra en relación a la satisfacción con el producto, la lealtad y el comportamiento de queja. Otros comportamientos posteriores a la compra, relacionados con la separación del consumidor de los productos, tales como el reciclaje o la disposición, en general han recibido poca atención (Roster, 2001; Arnould \& Thompson, 2005; Saunders 2010; Ture 2011).

La disposición es una compleja forma de comportamiento, cuya característica principal el consumidor decidio dejar de usar (o nunca utilizar) un producto de su propiedad y que aún es completamente utilizable (Jacoby, Berning, \& Dietvorst, 1977). De esta forma, mientras que en el reciclaje, la utilidad principal del producto se ha agotado (Belk, Sherry, \& Wallendorf, 1988; Paden \& Stell, 2005) y sus restos se reorientan a nuevos procesos de fabricación; en su disposición la utilidad principal del producto está todavía vigente, sin embargo los gustos, necesidades o condiciones de vida del propietario han cambiado (Hanson, 1980; Paden \& Stell, 2005).

El estudio de la disposición del consumidor es importante para el marketing debido a su capacidad predilecta de nuevas compras (Haws, Naylor, Bearden, \& Coulter, 2008) y al impacto en el posicionamiento de las marcas por el flujo de productos hacia nuevos propietarios (Harrell \& McConocha, 1992). El estudio de la disposición también es importante desde la perspectiva ecológica, pues el método utilizado por los consumidores determinará el tiempo de circulación de los productos y su llegada final a los botaderos de basura. Adicionalmente existe un interés también social y desde la psicología, por el impacto de prácticas extremas como la acumulación compulsiva (Haws, Naylor, Coulter, \& Bearden, 2012).

La disposición es un proceso de separación tanto física como sicológica de un objeto (Young, 1991b; Roster, 2001) que implica cuatro etapas (Hanson, 1980): Reconocimiento del problema o motivación para la disposición, la búsqueda y evaluación de alternativas, la selección del método de disposición y los resultados posteriores a la decisión.

El presente estudio se centró en las diferencias de género en diversas etapas del proceso de disposición de productos, busca contribuir y ampliar el conocimiento del comportamiento del consumidor en esta área descuidada de la investigación.

Revista Brasileira de Marketing - REMark, São Paulo, v. 12, n. 2, p. 158-179, abr./jun. 2013. 


\subsection{EL ESCENARIO DE ESTUDIO: ECUADOR, COLECTIVISMO Y ALTA MASCULINIDAD}

La investigación se realizó en Ecuador, un país latinoamericano en vías de desarrollo con una población superior a los 14 millones de habitantes. Dos características culturales importantes del Ecuador, son su bajo individualismo (alto colectivismo) y su alta masculinidad. Ecuador presenta

un índice de individualismo de 8\%, en el otro extremo de los Estados Unidos que es de 91\%; en cuanto al índice de masculinidad, este es de 63\%, muy cercano al de Estados Unidos que es de $62 \%$ (Hofstede, 2001).

En base a estas características, Ecuador puede concebirse como un país donde los individuos y sus objetivos están subordinados a los grupos que integran. Además los roles de género están muy bien demarcados, orientando a los hombres a la ejecución y al logro material, en temas de consumo se centra en las principales decisiones; mientras que las mujeres se orientan al cuidado de las personas y las relaciones, en materia de consumo a las decisiones rutinarias (Hofstede, 2001).

\section{MARCO TEÓRICO Y PROBLEMA DE INVESTIGACIÓN}

\subsection{GÉNERO Y COMPORTAMIENTO DEL CONSUMIDOR}

El género es una de las variables más usadas en el comportamiento del consumidor, siendo otros constructos alternativos a la misma, la identidad y las actitudes a los roles de género. Mientras el género hace referencia a la construcción social en torno al sexo biológico del consumidor (Marini, 1990), la identidad de género se centra en los rasgos de personalidad relacionados con masculinidad y feminidad, y las actitudes hacia los roles de género, que se centran en las actitudes hacia los papeles de la sociedad que prescribe para los hombres y las mujeres (Fischer \& Arnold, 1994). Por la fuerte carga cultural de la variable del género, en los estudios de comportamiento del consumidor, hombres y mujeres son tratados como subculturas (Schiffman, Kanuk, \& Wisenblit, 2010).

En las sociedades tradicionalistas se espera que los hombres sean más agresivos y competitivos y den el soporte económico al hogar, mientras se espera que las mujeres sean más delicadas y prudentes y se ocupen de las actividades al interior del hogar (Marini, 1990; Hofstede, 2001). Aun cuando en la mayoría de la sociedad se están produciendo cambios económicos y

Revista Brasileira de Marketing - REMark, São Paulo, v. 12, n. 2, p. 158-179, abr.jun. 2013. 
culturales acelerados, las mujeres aún son las principales responsables de las tareas del hogar (Gentry, Commuri, \& Jun, 2003). De hecho, en Ecuador y Latinoamérica, las tareas del cuidado de otros y de labores domésticas, son una seria barrera a la inserción de la mujer en el mercado laboral y si bien en la región, en el período 1999 al 2011, se observa una reducción de la pobreza e indigencia, el porcentaje de hogares indigentes y pobres encabezados por mujeres aumentó (CEPAL, 2012).

En materia al comportamiento del consumidor, los estudios revelan que las mujeres invierten más tiempo que los hombres en compras, pues reúnen más información sobre las alternativas disponibles, los precios y la composición de los productos (Cleveland, Babin, Laroche, Ward \& Bergeron, 2003), por lo que gastan menos dinero y satisfacen mejor los gustos y necesidades de los demás (Fischer \& Arnold, 1990). En la sociedad de alta masculinidad, los hombres realizan las principales decisiones de compra, mientras que las mujeres realizan las compras rutinarias, principalmente relacionadas con comida y artículos para el hogar (Hofstede, 2001).

\subsection{MOTIVACIONES PARA LA DISPOSICIÓN}

Las posibles motivaciones o razones que puede tener un consumidor para disponer productos, se encuentran fragmentadas y dispersas en estudios muy diversos, consecuencia de la también diversidad de productos que el consumidor dispone. Una primera razón es que los productos son insatisfactorios desde el inicio y el principal origen de ellos son los regalos inadecuados (Sherry, 1983; Rucker, Balch, Higham, \& Schenter, 1992; Sherry, McGrath, \& Levy, 1992), de esta forma los productos no son elegidos por el consumidor sino por los dadores.

Otras razones se congregan en torno a los cambios en la vida de los consumidores. Tanto los cambios físicos en el cuerpo (Schewe, 1988; Schewe \& Meredith, 1994), como cambios en las etapas de la vida y en la imagen del consumidor (Belk, 1988; Young, 1991b; Roster, 2001; Lastovicka \& Fernández, 2005) pueden llevar a la incompatibilidad entre los productos poseídos y

los nuevos requerimientos del consumidor. Adicionalmente, el consumidor puede desear alejar productos relacionados a momentos o etapas de triste recordación (Lastovicka \& Fernández, 2005).

Otros cambios relacionados a los ingresos del consumidor o a sus gustos y preferencias, pueden llevar a remplazar el producto actual por uno de diferente calidad (Jacoby et al., 1977; Morgan \& Birtwistle, 2009).

El entorno físico del consumidor puede ser otro factor en la disposición. El consumidor puede llegar a acumular una gran cantidad de productos, su familia puede crecer o simplemente

Revista Brasileira de Marketing - REMark, São Paulo, v. 12, n. 2, p. 158-179, abr.jun. 2013. 
puede mudarse a un hogar más pequeño; en todos estos casos aparece el problema del espacio físico disponible (Hanson, 1980; Harrell \& McConocha, 1992).

La búsqueda de variedad, es una característica psicológica que implica en que el consumidor ansíe una mayor estimulación a través de la diversidad en información, productos o marcas (Hirschman, 1980; Menon \& Kahn, 1995; Ratner, Kahn \& Kahneman, 1999) puede ser una causa adicional para la disposición de productos.

Cambios en el entorno cultural o tecnológico también pueden precipitar la disposición; así, los cambios de la moda llevan a que el producto se vea anticuado (Hanson, 1980; Morgan y Birtwistle, 2009) y a su vez los cambios tecnológicos producen la obsolescencia del producto poseído por el consumidor (Jacoby et al., 1977; Hanson, 1980; Okada, 2001).

Finalmente otra causa para la disposición está relacionada a la transitoriedad en el uso del producto. El consumidor puede comprar productos pensando sólo en unas ocasiones de uso específicas, luego de las cuales se vuelve innecesaria (Morgan \& Birtwistle, 2009).

El tema género del consumidor y la motivación para la disposición, no ha sido abordado integralmente por los estudios revisados. Dichos estudios han abordado el problema desde una óptica más bien especializada y en la mayoría de los casos sin interés de asociar el género y la disposición.

\subsection{FORMAS DE DISPOSICIÓN}

La tipología más conocida de métodos de disposición fue generada por Jacoby et al. (1977), quien planteó que un consumidor tiene las siguientes opciones para disponer un producto: Mantener el producto (convertirlo a otro uso o almacenarlo), disponerlo de manera temporal (rentarlo o prestarlo) y disponer de forma permanente (arrojarlo, darlo a otros, venderlo o negociarlo).

En cuanto a la relación de las formas de disposición y de género, dos estudios han afrontado este problema. Harrell \& McConocha (1992) en su investigación sobre intenciones de comportamiento de disposición y factores personales (donde el género fue uno de ellos), encontraron que era más probable el comportamiento de los hombres con respecto al de las mujeres, los hombres se comportan seleccionando y conservando el producto, y las mujeres respecto a los hombres donando sin deducción de impuestos; sin embargo no encontraron relación entre el género y regalar. Por otro lado, Young (1991a) en base a una investigación en la cual pedía a cada participante recordar disposiciones de posesiones que cumplieron ciertas características, se encontró que las mujeres tenían una mayor tendencia a recordar disposiciones relacionadas con dar a otros y los hombres con destrucción involuntaria.

Revista Brasileira de Marketing - REMark, São Paulo, v. 12, n. 2, p. 158-179, abr./jun. 2013. 
Estos estudios tienen limitaciones que ameritan investigación adicional. En el estudio de Harrell \& McConocha (1992), a los encuestados se los colocó en una situación hipotética y frente a un estímulo tan general como imaginar un producto de "valor moderado", para luego pedirles que indiquen sus tendencias de comportamiento siguiendo cada método de disposición, adicionalmente se centraron solo en los "planificadores", es decir las personas más involucradas en la disposición, que en su mayoría fueron mujeres. En el caso de Young (1991a), su investigación estuvo centrada en las disposiciones memorables, que por lo mismo son situaciones inusuales y extremas.

De esta forma, ninguno de los dos estudios se centró en lo que se podría llamar el comportamiento real y cotidiano de los consumidores respecto al tema de disposición, investigación que en el actual estado del conocimiento se vuelve imprescindible (Saunders, 2010).

\subsection{PROBLEMA DE INVESTIGACIÓN E HIPÓTESIS}

Dentro del proceso de toma de decisiones de disposición, dos aspectos fueron de central importancia para el presente estudio sobre las diferencias de género: la motivación y la decisión de disposición; respecto a una categoría de productos en específico, las prendas de vestir. Esta categoría de producto fue seleccionado tanto por su característica de semi durable, como por su importancia en la vida de los consumidores en cualquier rango de edades e ingresos.

Ningún estudio conocido ha tratado integralmente el tema del género y la motivación para la disposición, sin embargo por la revisión de la literatura, se podría esperar que en el entorno cultural estudiado, las mujeres sean más conscientes de problemas de espacio en el hogar y de las necesidades de otros; adicionalmente, está bien establecido que las mujeres son más innovadoras en la moda de vestimenta que los hombres (e.g. Goldsmith, Stith, \& White, 1987; Stith \& Goldsmith, 1989).

Estos razonamientos llevan a plantear la siguiente hipótesis, para el escenario estudiado:

H1 - El género del consumidor está asociado con la motivación o las razones para disponer prendas de vestir.

Harrell \& McConocha (1992), encontraron una mayor tendencia con las mujeres respecto a los hombres a ser planificadoras en la disposición y a seleccionar el comportamiento de donar sin deducción, mientras que los hombres a conservar. Young (1991a), por su parte encontró que las disposiciones memorables para las mujeres implicaron dar a otros. Desde este conocimiento de partida, se esperaría en el entorno cultural analizado, un fuerte impacto del género en la disposición, prefiriendo las mujeres (a diferencia de los hombres) métodos que impliquen el cuidado de los demás y de las relaciones, como donar o regalar.

Revista Brasileira de Marketing - REMark, São Paulo, v. 12, n. 2, p. 158-179, abr.jun. 2013. 
En base a estos razonamientos se plantea la siguiente hipótesis para el escenario estudiado:

H2 - El género del consumidor está asociado con la forma o método en que esta dispuesta la vestimenta.

\section{METODOLOGÍA}

La investigación se realizó en Quito, capital de Ecuador, mediante la aplicación de un cuestionario a personas que concurren a una universidad privada en la modalidad semipresencial, es decir los sábados cada quince días. Quienes se inscriben en este tipo de programas buscan, ya sea terminar sus carreras de pre graduación o tomar cursos de formación continua, lo que hace que sus características demográficas y socioeconómicas sean muy diversas; teniendo en común el hecho de que todos tienen trabajos a tiempo completo y buscan gran flexibilidad en sus estudios.

Quito tiene una población alrededor de 2 millones de habitantes y representa el $87 \%$ de la población de la provincia de Pichincha. La tabla 1 muestra las características de la muestra, comparadas con las de la población adulta de la provincia de Pichincha. En promedio la muestra resultó un tanto más joven, con más nivel educativo y con más ingresos que la población.

Tabla 1 - Comparación entre las características de la muestra y de la población

\begin{tabular}{|c|c|c|}
\hline VARIABLE & MUESTRA & $\begin{array}{c}\text { POBLACIÓN ADULTA DE } \\
\text { PICHINCHA (18 AÑOS O MÁS) }\end{array}$ \\
\hline Edad promedio & 33 (rango de 18 a 60 años) & $36^{*}$ \\
\hline Años promedio de estudio & 12 & $10^{*}$ \\
\hline Ingresos mensuales promedio & \$US 704 (rango de \$200 a \$3500) & \$US $607^{* *}$ \\
\hline
\end{tabular}

*INEC (Instituto Nacional de Estadísticas y Censos)

$* *$ IPSA

La encuesta fue aplicada en el mes de octubre del 2011. Para el estudio de las diferencias de género en la motivación de la disposición, se usaron las categorías generadas en la revisión de la

literatura, se exceptuó la obsolescencia tecnológica por no ser relevante para la disposición de la vestimenta.

Para el estudio de las diferencias de género en los métodos de disposición, se utilizó la tipología de Jacoby et al. (1977) con algunas modificaciones. A la categoría de "dar" se la dividió en regalar y donar, división usual en los estudios de disposición (e.g. Harrell \& McConocha, 1992; Morgan \& Birtwistle, 2009); de esta forma, mientras que regalar está asociado con dar el producto a 
familiares, amigos y conocidos con los que se tiene un nexo; donar se asocia con dar a desconocidos u organizaciones de caridad. A la categoría de "negociar" se le convirtió en "intercambiar", término más usual en el entorno investigado y puesto que ya existía una categoría para vender. Adicionalmente se agregó una nueva categoría encontrada en estudios anteriores, que fue destruir el producto (Sherry et al., 1992).

El cuestionario comenzó explicando a los encuestados lo que significa disponer y preguntando sobre la última vez que decidieron disponer una prenda de vestir exterior (debían elegir una sola); además se pedía que si no habían tomado esta decisión o no recordaban los detalles, se abstengan de participar.

La fase de prueba del cuestionario se hizo con una muestra de 50 estudiantes y llevó a añadir a las motivaciones encontradas en la revisión de la literatura, dos opciones más: Alguien cercano al consumidor le dijo que deje de usar el producto ya alguien necesitaba el producto más que el consumidor.

Para la fase definitiva, el cuestionario fue aplicado de forma auto administrada a las personas que se encontraban realizando sus cursos. Se repartieron 700 cuestionarios y se recibieron llenos 438 de los cuales 414 estaban utilizables, 202 procedieron de hombres (48.8\%) y 212 de mujeres (51.2\%). El cuestionario consistió en 15 preguntas y aproximadamente tomó a los encuestados 15 minutos completarlo. Para los análisis de la asociación entre el género y la motivación para la disposición y entre el género y las formas de disposición, se utilizó el método de tabulación cruzada y el paquete estadístico IBM-SPSS $®$.

\section{RESULTADOS}

Las prendas de vestir que los encuestados reportaron haber utilizado por última vez fueron diversas, destacándose por su frecuencia los pantalones $(27.1 \%)$, los zapatos (18.1\%), las camisas o blusas (15.9\%) y las chaquetas, abrigos o chompas (13.3\%). Adicionalmente los artículos tuvieron precios que fluctuaron entre US\$3 a US\$250, teniendo en promedio un precio de US\$33.8.

\subsection{GÉNERO Y MOTIVACIÓN PARA LA DISPOSICIÓN}

En la muestra analizada, las principales razones para la disposición (tabla 2), fueron que el producto ya no quedaba físicamente bien al consumidor (34.8\%), que el producto ya no refleja la imagen que el consumidor deseaba proyectar (13.3\%), que el producto ya no estaba a la moda $(13.0 \%)$ y que fue un regalo insatisfactorio $(10.1 \%)$.

Revista Brasileira de Marketing - REMark, São Paulo, v. 12, n. 2, p. 158-179, abr.jun. 2013. 
Tabla 2 - Relación entre el género y la motivación para la disposición

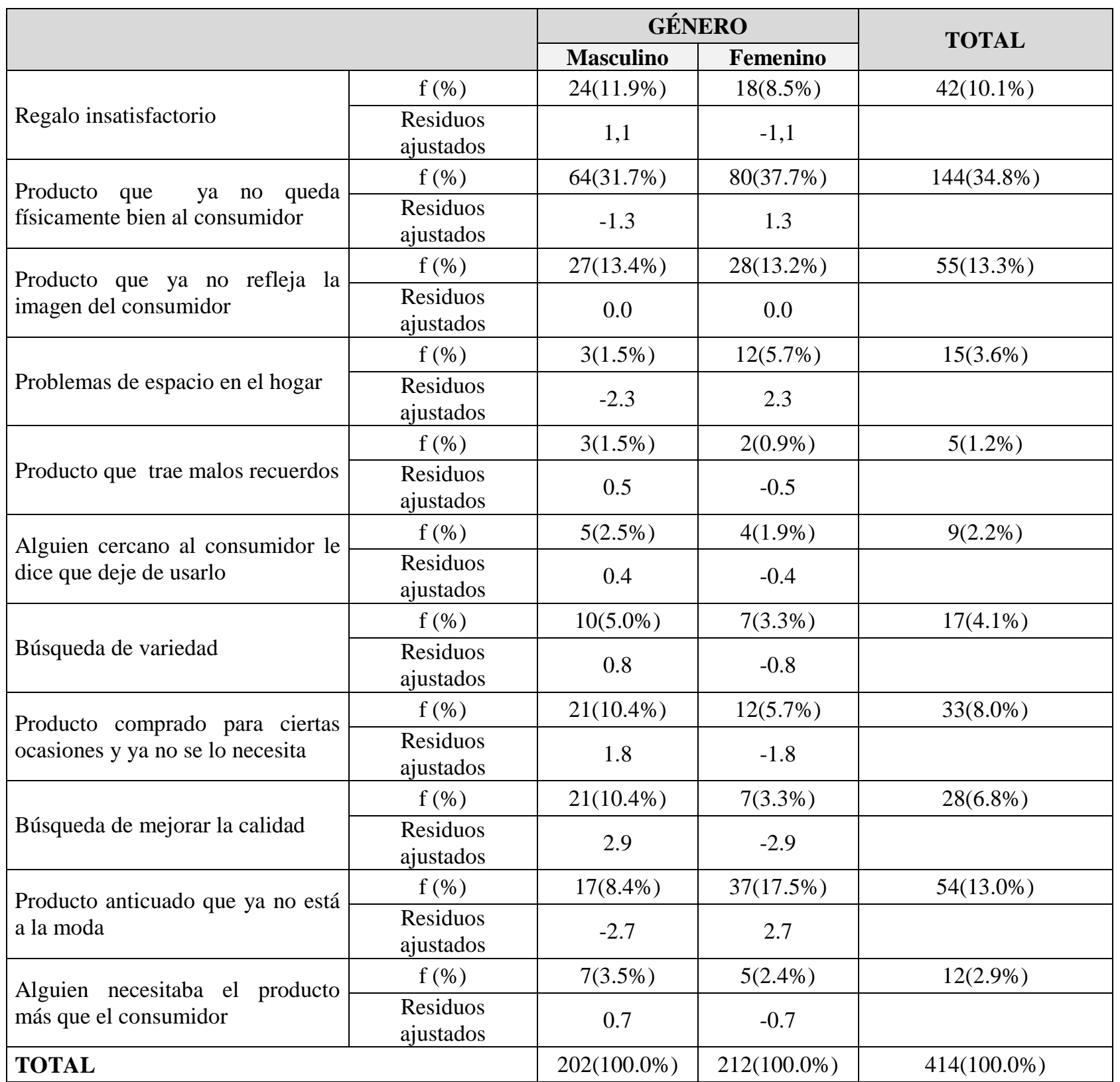

En la prueba chi cuadrado se obtuvo 4 celdas (18.2\%) con conteos esperados menores a 5 y el conteo mínimo esperado fue de 2.44, por lo que se satisfacía las recomendaciones de Cochran (1954), respecto a que para tablas con más de un grado de libertad, es recomendable, que un porcentaje no mayor del $20 \%$ de los conteos esperados sean menores a 5 y que todos deberían ser mayores a 1 .

Los resultados de la prueba chi cuadrado x2 $(10, \mathrm{~N}=414)=25.862, \mathrm{p}<0.01$, llevaron al rechazo de la hipótesis nula o de independencia y a apoyar la hipótesis de investigación respecto a que el género del consumidor está asociado con la motivación para disponer la vestimenta. Para el cálculo del tamaño del efecto se obtuvo una V de Cramer=0.250, lo que significa un grado de asociación moderado (Cohen 1992; Rea \& Parker 2005).

Revista Brasileira de Marketing - REMark, São Paulo, v. 12, n. 2, p. 158-179, abr.jun. 2013. 
Una vez comprobada la dependencia de las variables, para el análisis post hoc, se obtuvo los residuos ajustados (Haberman, 1973), a fin de identificar las celdas que contribuyeron de manera significativa a la dependencia de las variables. De este análisis se puede afirmar que la asociación entre el género y la motivación para la disposición, se debió principalmente a motivaciones como "problemas de espacio en el hogar", "búsqueda de mejorar la calidad" y "producto anticuado que ya no está a la moda" (ver tabla 2).

Las mujeres de la muestra tuvieron una tendencia significativamente mayor y los hombres menor a lo esperado, bajo condiciones de independencia a disponer por problemas de espacio en el hogar $(\mathrm{p}<0.05)$, o por no estar el producto a la moda $(\mathrm{p}<0.01)$. Por otro lado los hombres de la muestra tuvieron una propensión significativamente mayor y las mujeres menor a lo esperado bajo condiciones de independencia a disponer para mejorar la calidad del producto $(\mathrm{p}<0.01)$.

\subsection{GÉNERO Y FORMAS DE DISPOSICIÓN}

Un primer asunto interesante es que los hombres de la muestra tuvieron una tendencia significativamente menor y las mujeres mayor de lo que se esperaría en condiciones de independencia, a tomar la decisión de la forma de disposición por ellos mismos, $\mathrm{x} 2(1, \mathrm{~N}=414)=$ 9.246, p<0.01. De esta forma, 171 hombres (84,7\% del total de hombres) y 199 mujeres (93,9\% del total de mujeres) tomaron dicha decisión. Con estos 370 casos se realizó el análisis de la relación entre el género y las formas de disposición.

La aplicación de las formas de disposición de Jacoby et al. (1977), dio como resultado que 8 celdas (50.0\%) tuvieron conteos esperados menores a 5 y el conteo esperado mínimo fuera de 0.46. Las opciones "prestar" y "rentar" no se consideraron pues ningún participante las usó, lo que

refleja el carácter personal del uso de prendas de vestir. En estas condiciones la prueba chi cuadrado sustentaba la hipótesis de investigación, $x 2(7, \mathrm{~N}=370)=36.645, \mathrm{p}<0.001$.

A fin de adaptar los datos a las recomendaciones de Cochran (1954) sobre valores esperados mínimos, se procedió a colapsar categorías, cuidando conservar la homogeneidad (Shields \& Heeler, 1979). Para ello se utilizó los siguientes criterios:

Se colapsó las opciones "botar a la basura" con "destruir" en la categoría "deshacerse arrojando a la basura o destruyendo", categoría cuyo carácter esencial es la disposición que busca que nadie más, incluyendo el propietario obtenga un beneficio. Las opciones "intercambiar por otra cosa" y "vender", se colapsaron en la categoría denominada "beneficiarse con venta o intercambio", donde la esencia fue obtener un beneficio personal en la disposición. Las otras categorías permanecieron en su estado original.

Revista Brasileira de Marketing - REMark, São Paulo, v. 12, n. 2, p. 158-179, abr.jun. 2013. 
Tabla 3 - Relación entre el género y las formas de disposición

\begin{tabular}{|c|c|c|c|c|}
\hline & \multicolumn{2}{|c|}{ GÉNERO } & \multirow{2}{*}{ TOTAL } \\
\hline & & Masculino & Femenino & \\
\hline \multirow{2}{*}{ Usar para otros propósitos } & $\mathrm{f}(\%)$ & $14(8.2 \%)$ & $6(3.0 \%)$ & $20(5.4 \%)$ \\
\hline & Residuos ajustados & 2.2 & -2.2 & \\
\hline \multirow{2}{*}{ Guardar o almacenar } & $\mathrm{f}(\%)$ & $69(40.4 \%)$ & $44(22.1 \%)$ & $113(30.5 \%)$ \\
\hline & Residuos ajustados & 3.8 & -3.8 & \\
\hline \multirow{2}{*}{$\begin{array}{l}\text { Deshacerse arrojandolo a la } \\
\text { basura o destruyendolo }\end{array}$} & $\mathrm{f}(\%)$ & $9(5.3 \%)$ & $5(2.5 \%)$ & $14(3.8 \%)$ \\
\hline & Residuos ajustados & 1.4 & -1.4 & \\
\hline \multirow{2}{*}{ Regalar } & $\mathrm{f}(\%)$ & $63(36.8 \%)$ & $101(50.8 \%)$ & $164(44.3 \%)$ \\
\hline & Residuos ajustados & -2.7 & 2.7 & \\
\hline \multirow{2}{*}{$\begin{array}{l}\text { Beneficiarse con venta o } \\
\text { intercambio }\end{array}$} & $\mathrm{f}(\%)$ & $2(1.2 \%)$ & $3(1.5 \%)$ & $5(1.4 \%)$ \\
\hline & Residuos ajustados & -0.3 & 0.3 & \\
\hline \multirow{2}{*}{ Donar } & $\mathrm{f}(\%)$ & $14(8.2 \%)$ & $40(20.1 \%)$ & $54(14.6 \%)$ \\
\hline & Residuos ajustados & -3.2 & 3.2 & \\
\hline \multicolumn{2}{|l|}{ TOTAL } & $171(100.0 \%)$ & $199(100.0 \%)$ & $370(100.0 \%)$ \\
\hline
\end{tabular}

Bajo la nueva forma colapsada de la tabla 3, se obtuvo 2 celdas (16.7\%) con conteos esperados menores a $5 \mathrm{y}$ el conteo esperado mínimo fue de 2.31 . La prueba chi cuadrado x2 (5, $\mathrm{N}=370)=29.477$, p<0.001, llevó a rechazar la hipótesis nula de independencia y a apoyar la hipótesis de investigación, es decir, que existe una relación estadísticamente significativa entre el género del consumidor y la forma de disposición de las prendas de vestir. Al analizar el tamaño del efecto, se obtuvo una asociación moderada con una V de Cramer=0.282 (Cohen 1992; Rea y Parker 2005).

El análisis de los residuos ajustados (Haberman, 1973) permitió establecer que "usar para otros propósitos", "guardar o almacenar", "regalar" y "donar" contribuyeron significativamente a la asociación entre el género y las formas de disposición. Así, los hombres tuvieron una tendencia significativamente mayor y las mujeres menor, de lo que se habría esperado bajo condiciones de independencia, a disponer usando para otros propósitos $(\mathrm{p}<0.05)$ y a disponer guardando o almacenando $(\mathrm{p}<0.001)$. El porcentaje de mujeres que decidió disponer dando a otros ya sea

regalando o donando, fue significativamente mayor y el de los hombres significativamente menor $(\mathrm{p}<0.01)$ de lo que se habría esperado en condiciones de independencia. 


\subsection{GÉNERO, FORMAS DE DISPOSICIÓN Y OTRAS VARIABLES DEMOGRÁFICAS}

Para evaluar la asociación del género y las formas de disposición, cuando éste es controlado por la presencia de otras variables demográficas importantes, como la edad y el ingreso personal, se calculó un modelo de regresión logística multi nominal. Se escogió este procedimiento, puesto que para modelos multi variantes cuya variable dependiente es nominal, los métodos más apropiados son los logit (Long, 1997).

Los cálculos se realizaron usando el paquete estadístico IBM-SPSS ${ }^{\circ}$ y para ello se ingresaron como variables al género, la edad y el ingreso personal mensual. Al género por ser una variable categórica se le incorporó al modelo usando como variable de diseño género mujer (género hombre fue el parámetro de comparación). Al ingreso personal mensual se le dividió por 100 para evitar ratios grandes entre las desviaciones estándar de las variables incorporadas. En la variable dependiente, formas de disposición, se usó como categoría de referencia "guardar/almacenar".

Tabla 4 - Regresión logística multi nominal de la relación entre el género y las formas de disposición en presencia de otras variables demográficas.

\begin{tabular}{|c|c|c|}
\hline & & $\mathbf{B}(\mathbf{E E})$ \\
\hline \multirow{4}{*}{ Usar para otros propósitos } & Interceptó & $-2.62(0.99) * *$ \\
\hline & Edad & $.06(0.03)$ \\
\hline & Ingreso personal mensual & $-.10(0.08)$ \\
\hline & Género Mujer & $-.74(0.58)$ \\
\hline \multirow{4}{*}{$\begin{array}{l}\text { Deshacerse arrojandolo a la } \\
\text { basura o destruyendolo }\end{array}$} & Interceptó & $.28(1.33)$ \\
\hline & Edad & $-.06(0.05)$ \\
\hline & Ingreso personal mensual & $-.11(0.11)$ \\
\hline & Género Mujer & $-.27(0.63)$ \\
\hline \multirow{4}{*}{ Regalar } & Interceptó & $-1.42(0.54)^{* *}$ \\
\hline & Edad & $.03(0.02)$ \\
\hline & Ingreso personal mensual & $.04(0.03)$ \\
\hline & Género Mujer & $1.00(0.27)^{* * *}$ \\
\hline \multirow{4}{*}{$\begin{array}{l}\text { Beneficiarse con venta } 0 \\
\text { intercambio }\end{array}$} & Interceptó & $-1.61(2.34)$ \\
\hline & Edad & $-.12(0.09)$ \\
\hline & Ingreso personal mensual & $.16(0.08)$ \\
\hline & Género Mujer & $1.41(1.02)$ \\
\hline \multirow{4}{*}{ Donar } & Interceptó & $-4.36(0.79)^{* * *}$ \\
\hline & Edad & $.07(0.02)^{*}$ \\
\hline & Ingreso personal mensual & $.04(0.04)$ \\
\hline & Género Mujer & $1.56(.41)^{* * *}$ \\
\hline
\end{tabular}

Categoría de referencia: Guardar / almacenar

Nota: Modelo $\chi 2(15)=65.013, \mathrm{p}<0.001 .{ }^{*} \mathrm{p}<.05,{ }^{* *} \mathrm{p}<.01,{ }^{* * *} \mathrm{p}<.001$.

Como se puede observar en la tabla 4 , la presencia de la edad y el ingreso se alteró en cierta medida a la relación entre el género y las formas de disposición, pues ya no existieron diferencias 
de género significativas en disponer usando para otros propósitos. Sin embargo las diferencias de género persistieron en regalar, donar y guardar, los tres métodos de disposición más usuales en el entorno estudiado. Así, en presencia de la edad y el ingreso personal, las mujeres tuvieron una probabilidad significativamente mayor a los hombres tanto para regalar como donar (comparados con guardar); mientras que los hombres tuvieron una mayor probabilidad de guardar (comparada con regalar o donar). Por otro lado, el ingreso personal no fue un predictor significativo en ninguna relación y la edad predijo significativa y positivamente la probabilidad de donar.

\section{DISCUSIÓN}

El presente estudio abordó las diferencias de género en dos aspectos relevantes del proceso de toma de decisiones de disposición: la motivación y los métodos usados. En el primer aspecto es uno de los primeros estudios en afrontarlo integralmente, mientras que en respecto a los métodos de disposición, lo hizo de una forma muy diferente a otros estudios, centrándose en comportamientos de disposición reales y ordinarios.

Tanto la motivación para disponer prendas de vestir como la forma de hacerlo estuvieron significativamente relacionadas con el género de los participantes. A continuación se presenta una mayor discusión de los principales factores que contribuyeron a no apoyar las hipótesis de investigación.

\subsection{CAMBIOS EN LA VIDA Y SIMILITUDES DE GÉNERO EN LA DISPOSICIÓN}

Un primer tema de gran importancia son los cambios en las vidas de los consumidores. Estos cambios fueron los que originaron las motivaciones más frecuentes de disposición.

Los cambios físicos en el consumidor adulto se originan en corto plazo, en ganancias o pérdidas de peso y a largo plazo en el envejecimiento que trae consigo cambios en las proporciones corporales (Schewe, 1988; Schewe \& Meredith, 1994), estos cambios llevan a que el producto ya no presente un buen ajuste con el cuerpo; asociado con esto está el hecho de que el principal producto dispuesto fueron los pantalones $(27.1 \%)$. Como fuerza impulsora para la disposición, estos cambios afectaron de forma similar a hombres y mujeres.

Otros cambios importantes son aquellos en las etapas de la vida que conducen a asumir nuevos roles, por ejemplo en la edad adulta, la graduación, el inicio o finalización de relaciones significativas, los cambios de trabajo, el matrimonio, el divorcio, entre otros. Estos cambios llevan a

Revista Brasileira de Marketing - REMark, São Paulo, v. 12, n. 2, p. 158-179, abr./jun. 2013. 
que las posesiones anteriores no ayuden a desempeñar los nuevos roles y a proyectar la imagen deseada (Belk, 1988; Young, 1991b; Lastovicka \& Fernández, 2005), por ello son objeto de disposición. Nuevamente estos cambios afectaron de manera similar a hombres y mujeres en la disposición.

\subsection{ALTA MASCULINIDAD, EVOLUCIÓN Y DIFERENCIAS DE GÉNERO EN LA DISPOSICIÓN}

En la motivación para la disposición, un asunto muy interesante fue la tendencia significativamente mayor de las mujeres a disponer prendas de vestir por no estar a la moda y de los hombres por mejorar la calidad. Varios estudios han encontrado una mayor orientación de las mujeres a la moda de vestimenta, medida por una mayor conciencia, una adopción más temprana y un mayor gasto (e.g. Goldsmith et al., 1987; Gould \& Stern, 1989; Stith \& Goldsmith, 1989).

Desde la perspectiva de la "teoría de las estrategias sexuales humanas", los hombres y las mujeres han afrontado diferentes retos reproductivos a lo largo de su evolución, lo que lleva a las mujeres sentirse atraídas por los hombres que muestran capacidad para conseguir recursos valiosos y voluntad para invertirlos en ellas, mientras que los hombres se sienten atraídos por mujeres que exteriorizan juventud, salud y belleza (Buss, 1994). La moda sería desde esta perspectiva la forma en que las mujeres destacan su físico y muestran juventud y actualidad, mientras que las mejorías de la calidad en productos visibles serían formas en que los hombres demuestran la posesión de recursos valiosos.

Las mujeres de la muestra, presentaron una tendencia significativamente mayor que los hombres, a disponer por problemas de espacio en el hogar. Los roles de género en una sociedad de alta masculinidad ayudan a entender este comportamiento; en dichas sociedades, el hogar es el dominio de la mujer y es la que está más consciente de la distribución y uso del espacio del mismo.

En cuanto a los métodos de disposición, un asunto interesante fue la mayor propensión de los hombres frente a las mujeres, a disponer el producto dándole otros usos. Esta forma de

disposición se puede encasillar como "innovación de uso", la cual está influida por la “creatividad del consumidor" y los problemas de consumo que éste afronta (Hirschman, 1980). La creatividad no sería una explicación sustentable para las diferencias encontradas, en contraste con los problemas de consumo, que se muestran más promisorios.

En la división de tareas en una sociedad de alta masculinidad el hombre debe afrontar mayores problemas cuyas soluciones implican la reutilización de prendas de vestir; por ejemplo, para tareas en el hogar como arreglar el jardín, pintar la casa o lavar el carro. En el caso de las mujeres la reutilización de las prendas de vestir está más limitada.

Revista Brasileira de Marketing - REMark, São Paulo, v. 12, n. 2, p. 158-179, abr.jun. 2013. 
Adicionalmente es importante la tendencia mayor de los hombres llevar la frente a las mujeres, lo que se discute en la sección de implicaciones.

\subsection{COLECTIVISMO CULTURAL, SIMILITUDES Y DIFERENCIAS DE GÉNERO EN LA DISPOSICIÓN}

La principal forma de disposición que utilizaron los encuestados fue regalar el producto a familiares, amigos y conocidos (44.3\%), es decir utilizaron un canal de redistribución directo (Paden \& Stell, 2005) que demanda más esfuerzo del consumidor y que es reflejo del colectivismo, donde las personas estructuran sólidas redes interpersonales; la donación, es decir el flujo del producto fuera de estas redes, solo se dio en el 14,6\% de los casos. Por otro lado, los estudios en sociedades individualistas, han encontrado como principal elección a disponer, el donar (Harrell \& McConocha, 1992; Morgan \& Birtwistle, 2009), es decir utilizar un canal indirecto de redistribución (Paden \& Stell, 2005), que demanda menos esfuerzo del consumidor.

Es también llamativo el bajísimo impacto en el escenario estudiado, formas de disposición que llevan a deshacerse del producto de forma egoísta como arrojar a la basura o destruir (3.8\%) o a obtener réditos económicos como vender o intercambiar (1.4\%), porcentajes mucho menores a los reportados en la investigación de Harrell \& McConocha (1992).

En cuanto a las diferencias de género, las mujeres de la muestra presentaron una propensión significativamente mayor que los hombres a donar, lo que en general implica una mayor conciencia de las necesidades de los demás, en este caso, desconocidos. También mostraron una mayor tendencia a disponer por regalo, es decir dando a familiares y conocidos, lo que implica además de una mayor conciencia, un mayor conocimiento sobre dichas necesidades y sobre la reacción de los receptores ante este tipo de regalos. Las mujeres de manera predominante desarrollan las actividades de cuidado de las relaciones y de los nexos familiares (Rosenthal, 1985), lo que se traduce en un mayor dominio del canal de redistribución vía regalo o redistribución directa.

Revista Brasileira de Marketing - REMark, São Paulo, v. 12, n. 2, p. 158-179, abr./jun. 2013. 


\section{IMPLICACIONES Y LIMITACIONES}

El presente estudio usó una muestra relativamente grande y se centró en comportamientos reales y cotidianos sobre la disposición, desde una perspectiva integradora, lo que representa una fortaleza en el estado actual del conocimiento; sin embargo y a pesar de la cercanía de los estadísticos de la muestra con los parámetros poblacionales, la muestra no fue obtenida de forma aleatoria de la población, lo que es una limitación.

El presente estudio encontró una tendencia significativamente mayor de los hombres a guardar, siendo además el principal método de disposición de los mismos; esta situación amerita futuras investigaciones respecto a sus causas, que son diversas. Siendo una posible, el menor envolvimiento de los hombres respecto a las mujeres en la disposición; en este sentido, en la presente investigación, los hombres tuvieron una menor tendencia a tomar las decisiones de disposición por sí mismos y en la investigación de Harrell \& McConocha (1992) un porcentaje menor de hombres fueron "planificadores" en la disposición.

En el presente estudio se encontró que las diferencias de género persistieron en presencia de otras variables demográficas muy importantes como la edad y el ingreso personal. Un campo interesante para futuras investigaciones es la inclusión de más variables y sus interacciones, en la construcción de modelos de regresión logística multi nominal aplicados a la disposición. El género

es una primera candidata por los resultados obtenidos en el presente estudio; la edad del consumidor, el ingreso familiar, el nivel educativo y el precio del producto son otras candidatas obvias. Adicionalmente se puede incluir la conciencia ambiental y el comportamiento de reciclaje (Bianchi \& Birtwistle, 2012) y factores de estilo de vida como PRT o la tendencia a la retención del producto (Haws et al. 2012). Un punto muy importante al usar métodos de análisis como la regresión logística, es que el número de eventos mínimos por variable (EPV) debe ser de al menos 10 (Peduzzi, Concato, Kemper, Holford \& Feinstein, 1996); lo que demanda tomar muestras grandes relativamente inusuales de algunos métodos de disposición (en el presente caso, la venta e intercambio y el arrojar a la basura).

Si se considera que en la disposición, el producto conserva su capacidad de funcionamiento, una estrategia de marketing disponible para las empresas son los trade-ins, es decir la aceptación del producto dispuesto como parte de pago del nuevo, lo que además de atraer a los consumidores, de la empresa más control sobre la redistribución de los productos usados.

A este respecto y desde la perspectiva de la "contabilidad mental", Okada (2001) planteó que el costo mental (sicológico) de remplazar un producto todavía utilizable, está asociado con el valor que el consumidor considera aún no ha sido devengado por el producto (respecto al precio pagado por el mismo). Además encontró que los trade-ins, los regalos y las donaciones son formas

Revista Brasileira de Marketing - REMark, São Paulo, v. 12, n. 2, p. 158-179, abr.jun. 2013. 
competidoras para disminuir este costo psicológico; presentando los regalos superioridad sobre las donaciones y siendo además importantes moderadores de la eficacia de los trade-ins.

Junto a este conocimiento, al considerar uno de los resultados de la presente investigación, el regalo como principal método de disposición en el entorno colectivo estudiado y los resultados de otros estudios, respecto a la importancia de la donación en entornos individualistas (Harrell \& McConocha, 1992; Morgan \& Birtwistle, 2009); se puede esperar que en las sociedades colectivistas, los trade-ins aplicados a productos de valor moderado sean menos atractivos como estrategias promocionales, que en las sociedades individualistas. Si adicionalmente se considera la mayor tendencia general encontrada en las mujeres a regalar y de los hombres a guardar, también se puede esperar que los trade-ins como estrategia promocional, atraigan más a los hombres.

En el entorno colectivista estudiado, la principal forma de disposición usada por los encuestados fue regalar, es decir transferir el producto a miembros de su red social, lo que representa un canal de redistribución muy eficiente. Sin embargo, queda para las organizaciones de caridad un espacio importante de recolección, en la mayor tendencia de los hombres a guardar. La comodidad es básica si se parte del hecho que una de las razones para guardar es el menor interés de los hombres en la tarea de disposición.

Otro punto a considerar por las organizaciones no lucrativas es el atractivo porcentaje encontrado (10.1\%) de productos dispuestos procedentes de regalos insatisfactorios, donde hombres y mujeres se comportaron de forma relativamente similar y donde además no existe el efecto de valor remanente por devengar (por lo tanto se incrementaría el atractivo de donar frente a regalar). Las organizaciones de caridad pueden tener acceso a estos productos, pero evidentemente el momento indicado es después de las temporadas de regalos; en Occidente la Navidad es la mayor temporada de entrega de regalos.

Revista Brasileira de Marketing - REMark, São Paulo, v. 12, n. 2, p. 158-179, abr./jun. 2013. 


\section{REFERENCIAS}

Arnould, E. J. \& Thompson C. J. (2005). Consumer culture theory (CCT): Twenty years of research. Journal of Consumer Research, 31(4), 868-882.

Belk, R. W. (1988). Possessions and the extended self. Journal of Consumer Research, 15(2), 139168.

Belk, R. W.; Sherry J. F. y Wallendorf, M. (1988). A naturalistic inquiry into buyer and seller behavior at a swap meet. Journal of Consumer Research, 14(4), 449-470.

Bianchi, C. \& Birtwistle, G. (2012). Consumer clothing disposal behavior: A comparative study. International Journal of Consumer Studies, 36(3), 335-341.

Buss, D. M. (1994). The strategies of human mating. American Scientist, 82(3), 238-249.

Cleveland, M.; Babin, B.J.; Laroche, M.; Ward, P. \& Bergeron J. (2003). Information search patterns for gift purchases: A cross-national examination of gender differences. Journal of Consumer Behavior, 3(1), 20-47.

Cochran, W. G. (1954). Some methods for strengthening the common x2 tests. Biometrics, 10(4), 417-451.

Cohen, J. (1992). A power primer. Psychological Bulletin, 112(1), 155-159.

Comisión Económica para América Latina CEPAL (2012). Panorama social de América Latina. Recuperado en 25 de marzo, 2013, de http://www.eclac.org/publicaciones/xml/5/48455/PanoramaSocial2012DocI-Rev.pdf

Fischer, E. \& Arnold S.J. (1990). More than a labor of love: Gender roles and Christmas gift shopping. Journal of Consumer Research, 17(3), 333-345.

Fischer, E. \& Arnold, S.J. (1994). Sex, gender identity, gender role attitudes, and consumer behavior. Psychology \& Marketing, 11(2), 163-183.

Gentry, J.W.; Commuri, S. \& Jun, S. (2003). Review of literature of gender in the family. Academy of Marketing Science Review. 1, 1-18.

Goldsmith R. E., Stith, M. T. \& White, J. D. (1987). Race and sex differences in self-identified innovativeness and opinion leadership. Journal of Retailing, 63(4), 411-425.

Gould, S. J. \& Stern, B. B. (1989). Gender schema and fashion consciousness. Psychology \& Marketing, 6(2), 129-145.

Haberman, S. J. (1973). The analysis of residuals in cross-classified tables. Biometrics, 29(1), 205220.

Revista Brasileira de Marketing - REMark, São Paulo, v. 12, n. 2, p. 158-179, abr./jun. 2013. 
Hanson, J. W. (1980). A proposed paradigm for consumer product disposition processes. Journal of Consumer Affairs, 14(1), 49-67.

Harrell, G. D. \& McConocha, D. M. (1992). Personal factors related to consumer product disposal tendencies. Journal of Consumer Affairs, 26(2), 397-417.

Haws, K.; Naylor, R.; Bearden, W. O. \& Coulter, R. (2008). To have and to hold? Implications of disposition tendencies for consumer research. Advances in Consumer Research, 35, 764.

Haws, K.L.; Naylor, R.W.; Coulter, R.A. \& Bearden, W.O. (2012). Keeping it all without being buried alive: Understanding product retention tendency. Journal of Consumer Psychology, 22(2), 224-236.

Hirschman, E.C. (1980). Innovativeness, novelty seeking, and consumer creativity. Journal of Consumer Research, 7(3), 283-295.

Hofstede, G. (2001). Culture's consequences: Comparing values, behaviors, institutions and organizations across nations (2a ed.). Thousand Oaks, CA, USA: Sage Publications.

Instituto Nacional de Estadísticas y Censos del Ecuador INEC (2010). Base Censo 2010. Recuperado en 17 de marzo, 2012, de http://www.inec.gob.ec/cpv/index.php?option=com_content $\&$ view=article\&id=222\&Itemid=6 $6 \&$ lang=es.

IPSA Group Latin America. (2010). Ecuador overview 2010: Quito.

Jacoby, J.; Berning, C.K. \& Dietvorst, T.F. (1977). What about disposition? Journal of Marketing, 41(2), 22-28.

Lastovicka, J. L. \& Fernandez, K. V. (2005). Three paths to disposition: The movement of meaningful possessions to strangers. Journal of Consumer Research, 31(4), 813-823.

Long J.S. (1997). Regression models for categorical and limited dependent variables. Thousand Oaks, CA, USA: Sage Publications.

Marini, M.M. (1990). Sex and gender: What do we know? Sociological forum, 5(1), 95-120.

Menon, S. \& Kahn, B. E. (1995). The impact of context on variety seeking in product choices. Journal of Consumer Research, 22(3), 285-295.

Morgan, L. R. \& Birtwistle, G. (2009). An investigation of young fashion consumer's disposal habits. International Journal of Consumer Studies, 33(2), 190-198.

Okada, E. M. (2001). Trade-ins, mental accounting, and product replacement decisions. Journal of Consumer Research, 27(4), 443-446.

Revista Brasileira de Marketing - REMark, São Paulo, v. 12, n. 2, p. 158-179, abr./jun. 2013. 
Paden, N. \& Stell, R. (2005). Consumer product redistribution: Disposition decisions and channel options. Journal of Marketing Channels, 12(3), 105-123.

Peduzzi, P.; Concato, J.; Kemper, E.; Holford, T.R. \& Feinstein, A.R. (1996). A simulation of the number of events per variable in logistic regression analysis. Journal of Clinical Epidemiology, 49(12), 1373-1379.

Ratner, R. K.; Kahn, B. E. \& Kahneman, D. (1999). Choosing less-preferred experiences for the sake of variety. Journal of Consumer Research, 26(1), 1-15.

Rea, L. M. \& Parker, R. A. (2005). Designing and conducting survey research: A comprehensive guide ( 3 a ed.). San Francisco, CA, USA: Jossey Bass.

Rosenthal, C.J. (1985). Kinkeeping in the family division of labor. Journal of Marriage and the Family", 47(4), 965-974.

Roster, C. A. (2001). Letting go: The process and meaning of dispossession in the lives of consumers. Advances in Consumer Research, 28(1), 425-430.

Rucker, M; Balch, T.; Higham, F. \& Schenter, K. (1992). Thanks but no thanks: Rejection, possession and disposition of the failed gift. Advances in Consumer Research, 19, 488.

Saunders, S. G. (2010). An exploratory study into the disposition behaviour of poor bottom-of-thepyramid urban consumers. Advances in Consumer Research, 37, 440-446.

Schewe, C.D. (1988). Marketing to our aging population: Responding to physiological changes. Journal of Consumer Marketing, 5(3), 61-73.

Schewe, C. D. \& Meredith G. E. (1994). Digging deep to delight the mature adult consumer. Marketing Management, 3(3), 20-35.

Schiffman, L.G.; Kanuk, L.L. \& Wisenblit J. (2010). Consumer behavior (10th ed.). Upper Saddle River, NJ, USA: Pearson.

Sherry, John. F. Jr. (1983). Gift giving in anthropological perspective. Journal of Consumer Research, 10(2), 157-168.

Sherry, J. F. Jr.; McGrath, M.A. \& Levy, S. J. (1992). The disposition of the gift and many unhappy returns. Journal of Retailing, 68(1), 40-65.

Shields, W. S. \& Heeler, R. M. (1979). Analysis of contingency tables with sparse values. Journal of Marketing Research, 16(3), 382-386.

Stith, M.T. \& Goldsmith, R.E. (1989). Race, sex and fashion innovativeness: A replication. Psychology \& Marketing, 6(4), 249-262. 
Diferencias de Género en la Motivación y en los Métodos de Disposición de Productos en un Entorno Colectivista de Alta Masculinidad

Ture, M. (2011). Disposing: Constructing and structuring consumption. Advances in Consumer Research, 39, 32-35.

Young, M. M. (1991a). "Males, females, and possession disposition. Gender and Consumer Behavior, 1, 105-114.

Young, M. M. (1991b). Disposition of possessions during role transitions. Advances in Consumer Research, 18, 33-39.

Data do recebimento do artigo: 11/01/2013

Data do aceite de publicação: 15/04/2013

Revista Brasileira de Marketing - REMark, São Paulo, v. 12, n. 2, p. 158-179, abr./jun. 2013. 